\title{
Ascorbic acid: properties, determination and uses
}

\begin{abstract}
Vitamin $\mathrm{C}$ is one of the most important vitamins in human nutrition. However, the complexity of food matrices and the degradation of this vitamin pose huge challenges during analysis. The chemistry (spectral and physical properties), uses, and analytic methods for the determination of vitamin $\mathrm{C}$ are highlighted, along with the strategies used by various researchers to prevent vitamin oxidation during sample extraction. Furthermore, the advantages and disadvantages of different analytic methods, traditional and modern, are also discussed.
\end{abstract}

Keyword: Association of Official Analytical Chemists (AOAC); Capillary electrophoresis (CE); Chemiluminescence; Derivatization; Electrochemical (EC); High-performance liquid chromatography (HPLC); 1-Ascorbic acid (1-AA); 1-Dehydroascorbic acid (1-DHAA); Oxidation; Reduction; Sample extraction; Spectrophotometric; Stability 